\title{
Combining Polymers with the Functionality of Proteins: New Concepts for Atom Transfer Radical Polymerization, Nanoreactors and Damage Self-reporting Materials
}

\author{
Nico Bruns ${ }^{\star a b}$, Samuel Lörcher ${ }^{\mathrm{b}}$, Katarzyna Makyła ${ }^{\mathrm{b}}$, Jonas Pollard ${ }^{\mathrm{ab}}$, Kasper Rengglib, \\ and Mariana Spulber ${ }^{\mathrm{b}}$
}

\begin{abstract}
Proteins are macromolecules with a great diversity of functions. By combining these biomolecules with polymers, exciting opportunities for new concepts in polymer sciences arise. This highlight exemplifies the aforementioned with current research results of our group. We review our discovery that the proteins horseradish peroxidase and hemoglobin possess ATRPase activity, i.e. they catalyze atom transfer radical polymerizations. Moreover, a permeabilization method for polymersomes is presented, where the photoreaction of an $\alpha$-hydroxyalkylphenone with block copolymer vesicles yields enzyme-containing nanoreactors. A further intriguing possibility to obtain functional nanoreactors is to enclose a polymerization catalyst into the thermosome, a protein cage from the family of chaperonins. Last but not least, fluorescent proteins are discussed as mechanoresponsive molecular sensors that report microdamages within fiber-reinforced composite materials.
\end{abstract}

Keywords: Biotransformations · Enzyme-catalyzed atom transfer radical polymerization . Mechanoresponsive materials $\cdot$ Nanoreactors $\cdot$ Polymer-protein hybrid materials

\section{Introduction}

Polymers have clearly contributed to key advances in material science, nanotechnology, and in many day-to-day aspects of modern life. They are commonly used in biomedical applications, as lightweight materials for transportation, as packaging, or as building blocks for nanostructures. The properties of polymers can be tailored and fine-tuned to the requirements of specific applications, and polymers are easily produced both on laboratory scale and on large industrial scale. However, the functionality of polymers is still relatively simple and can hardly compete with the multifunctionality and complexity of natural materials in living organisms. For example, self-healing and self-sensing of damaged tissue are properties widely found in nature, while the development of synthetic materials with such properties is still an evolving field of research with ma-

\footnotetext{
${ }^{\star}$ Correspondence: Prof. Dr. N. Bruns ${ }^{\mathrm{a}}$

Tel.: +41263009254

E-mail: nico.bruns@unifr.ch

aAdolphe Merkle Institute

University of Fribourg

Route de l'ancienne Papeterie CP 209

$\mathrm{CH}-1723$ Marly 1

'Department of Chemistry

University of Basel

Klingelbergstrasse 80

$\mathrm{CH}-4056$ Basel
}

ny challenges to overcome. ${ }^{[1]}$ Functionality in natural materials is often hierarchically organized on various length scales, starting with molecular building blocks like proteins and DNA, to cells, tissues, and organisms. On the molecular level, proteins and their catalytically active variants, enzymes, are key contributors to the complexity and multifunctionality of life. Their catalytic, structural, sensing, responsive, and selfassembly properties are rarely matched by synthetic molecules such as polymers or catalysts. Therefore, we are interested in harvesting some of these functional properties to the benefit of polymer science. Here, we will highlight recent results on the use of enzymes as environmentally friendly catalysts for controlled radical polymerizations. ${ }^{[2]}$ Moreover, we will present work on polymeric and protein nanoreactors, ${ }^{[3]}$ and we will review protein-polymer hybrid materials with the capability of selfsensing microdamages. ${ }^{[4]}$

\section{ATRPases}

Atom transfer radical polymerization (ATRP) is one of the most important synthetic techniques in polymer chemistry of past decades. ${ }^{[5]}$ The steadily increasing number of research articles published on ATRP and related controlled/living radical polymerization techniques is a testimony of the synthetic value of these methods..$^{[5 c]}$ They allow the synthesis of well-defined polymers by controlling the molecular weight during synthesis while keeping the distribution of chain lengths, i.e. the polydispersity, to a minimum. Moreover, ATRP chemistry is tolerant to a variety of functional groups present in monomers, initiators, and reaction media. Furthermore, ATRP allows the insertion of functional end groups at polymer chain ends. It therefore is suited for the synthesis of polymers with complex architectures. All these synthetic peculiarities have enabled modern polymer chemistry to provide a multitude of functional macromolecular building blocks, e.g. for the bottom-up assembly of nanostructures, for the modification of surfaces, and for biomedical devices. However, ATRP requires the use of organometal-based catalysts, e.g. complexes of $\mathrm{Cu}(\mathrm{I}), \mathrm{Fe}(\mathrm{II})$, and $\mathrm{Ru}(\mathrm{II})$. In the ATRP equilibrium, these catalysts reversibly abstract a halogen atom from a halogenated organic compound, such as an appropriate initiator or a halogen-terminated polymer chain, thereby forming a radical at the initiator or the polymer chain end. Subsequently the polymerization reaction proceeds via a chain-growth mechanism until the halogen atom is transferred from the catalyst to the active chain end, thus forming a dormant species. This can be reactivated in a further 
reaction cycle. Importantly, the equilibrium between active and dormant polymer chain is strongly shifted to the dormant species, which ensures a low overall concentration of radicals throughout the reaction. Hence, radical termination reactions are suppressed and control over the polymerization is obtained. The use of these catalysts has some drawbacks, however, as they are tedious to remove from the product polymer. The catalysts can be toxic and therefore render the products incompatible with biomedical applications. Moreover, the catalysts can interfere with using the material in electronic applications or simply cause unwanted coloration of the final product. ${ }^{5 b, c]}$

Some of these disadvantages could be overcome if enzymes were to be used as catalysts in ATRP. Enzymes are derived from renewable resources, they are non-toxic and edible and can be easily separated from synthetic polymers, e.g. by precipitation or affinity filtration. In fact, enzymes are known to catalyze, mediate or promote almost all classes of polymerizations known in synthetic polymer chemistry, e.g. ring-opening polymerization, polycondensations, and ionic polymerizations. ${ }^{6]}$ Not surprisingly, biocatalytic polymerizations have found widespread interest and use. ${ }^{[6]}$ However, until recently no enzymatically catalyzed controlled/ living radical polymerizations were described in the literature, despite the many redox enzymes that are able to initiate free radical polymerization ${ }^{[6 c]}$ or are involved in dehalogenation or halogenation reactions. ${ }^{[7]}$ The reversible dehalogenation and rehalogenation of organic compounds, however, that would allow controlled/living radical polymerizations was unknown.

We discovered that the classic heme proteins horseradish peroxidase (HRP) and hemoglobin $(\mathrm{Hb})$ are able to catalyze the polymerization of vinyl monomers under ATRP conditions (Fig. 1A). ${ }^{[2]}$ Both biomacromolecules are promiscuous proteins with a variety of functions in vivo and in vitro. The main function of hemoglobin is its ability to bind and transport oxygen. Moreover, it is sometimes referred to as an honorary enzyme, ${ }^{[8]}$ because it is able to catalyze reactions involving hydrogen peroxide, i.e. it displays peroxidase activity. ${ }^{[9]}$ HRP is an enzyme found in the root of horseradish, which catalyzes single electron oxidations of, for example, aromatic substrates while consuming hydrogen peroxide. ${ }^{[10]} \mathrm{HRP}$ has been exploited as a work-horse enzyme in biotechnology and can be found e.g. in ELISA tests. Moreover, it has been intensively investigated as a mediator of free radical polymerizations.[6c,11] Both proteins contain heme as prosthetic group, i.e. iron protoporphyrin IX complexes. While hemoglobin is a tetrameric protein consisting of two $\alpha$-and two $\beta$-subunits, HRP is monomeric. Another pronounced difference between the two proteins is that the redox chemistry of hemoglobin involves mostly $\mathrm{Fe}(\mathrm{II})$ and $\mathrm{Fe}(\mathrm{III})$ redox states, while HRP's activity involves changes between $\mathrm{Fe}(\mathrm{III})$ and $\mathrm{Fe}(\mathrm{IV})$.

In our initial reaction, N-isopropylacrylamide (NIPAAm) was polymerized with $\mathrm{Hb}$ under activators regenerated by electron transfer (ARGET) ATRP conditions, i.e. in the presence of the reducing agent ascorbic acid.[2c] This reaction was meant to be a control reaction as we were investigating strategies to conjugate conventional ATRP catalysts to proteins. We did not expect to obtain any polymer with a native, non-modified protein. To our surprise polyNIPAAm formed and could be easily detected by its property to precipitate from aqueous solution upon heating above the lower critical solution temperature (LCST) of $33{ }^{\circ} \mathrm{C}$. The formation of polymer was further confirmed by ${ }^{1} \mathrm{H}-\mathrm{NMR}$. Taking these initial results as a starting point, the activity of $\mathrm{Hb}$ in these polymerizations was studied in detail. Hemoglobin has surface-accessible cysteines that interfere with radical polymerization. They can act as chain transfer agents. Thus, they were blocked with a maleimide reagent, yielding Cys-blocked Hb. This modified hemoglobin is able to form radicals from typical ATRP initiators, such as 2-hydroxyethyl2-bromoisobutyrate (HEBIB) and 2-bromopropionitrile (BPN). Therefore it can initiate polymerization of vinyl monomers. Moreover, bromine atoms are transferred back to the polymer radicals, as evidenced by the detection of bromine-terminated polymer chains in neutron activation analysis and by chain extension experiments. Therefore, the mechanism involved in this polymerization is closely related to the ATRP-mechanism with its reversible halogenation and dehalogenation reactions. The degree of control over the reaction depends on the monomer. Three monomers were investigated, NIPAAm, poly(ethylene glycol) methyl ether acrylate (PEGA) and poly(ethylene glycol) methyl ether methacrylate (PEGMA) (Fig. 1B and C). The polymerization of all three monomers
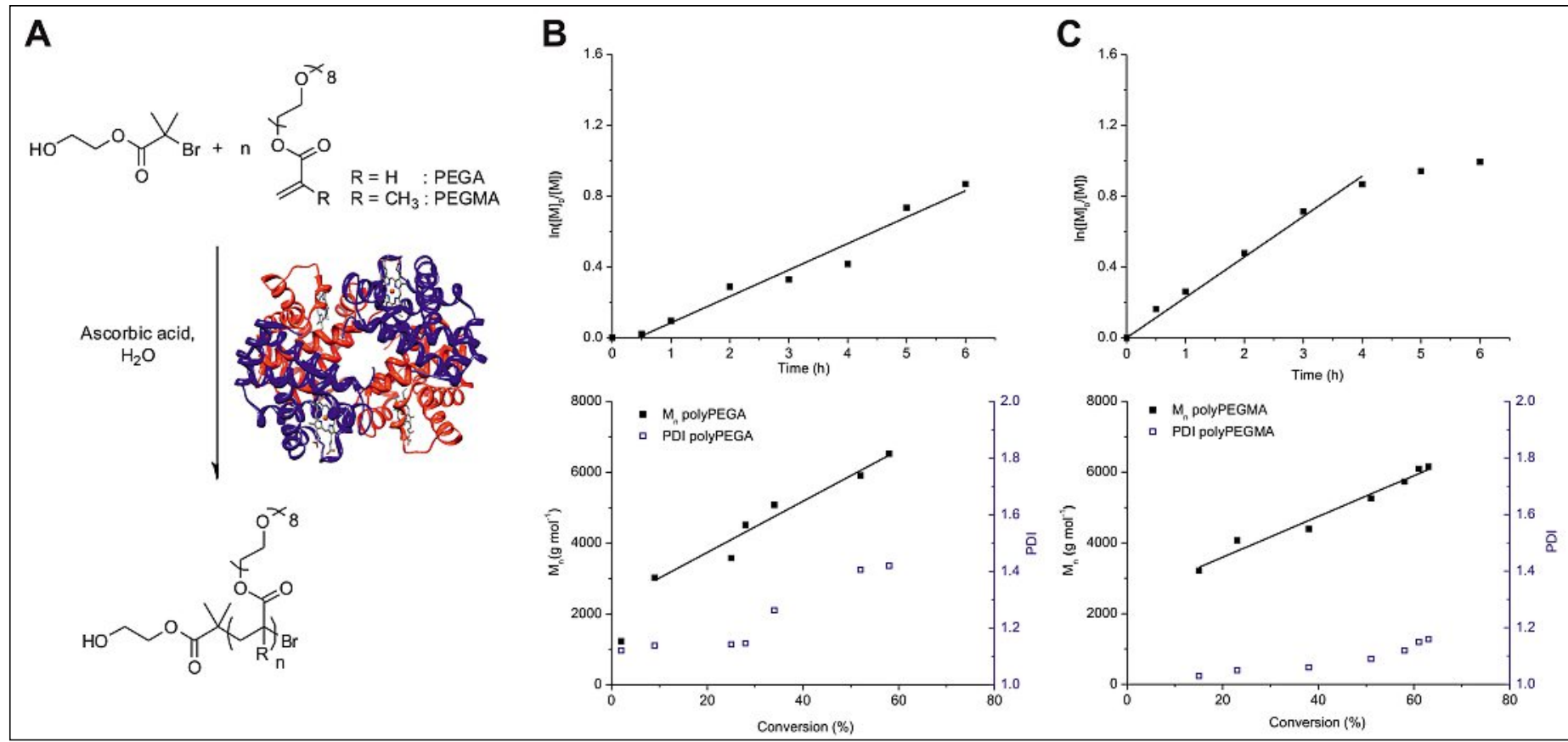

Fig. 1. A) ARGET ATRP of water-soluble monomers catalyzed by Cys-blocked hemoglobin. B, C) First order kinetic plots, and molecular weight and polydispersity as a function of conversion: (B) PEGA, (C) PEGMA. Adapted with permission from ref. [2c]. Copyright 2013 American Chemical Society. 
followed first order kinetics, indicating a constant radical concentration throughout the reaction. The molecular weights of polyPEGA and polyPEGMA did increase with conversion, while the polymerization of NIPAAm did not show this behavior. The polydispersity indices remained below 1.42 for PEGA and 1.17 for PEGMA. They showed a tendency to increase at conversions higher than $60 \%$. In conclusion, PEGA and PEGMA polymerizations were controlled with a reasonable, but not perfect degree of control.

Special emphasis was placed on the characterization of $\mathrm{Hb}$ throughout the biocatalytic ATRP process. Gel electrophoresis, circular dichroism (CD) spectroscopy, and UV-vis spectroscopy revealed that hemoglobin was stable throughout the reaction and only underwent minor conformational changes. The redox chemistry of the heme group during ATRP was investigated by time-resolved UV-vis spectroscopy and allowed to draw the following mechanistic picture. Commercially available hemoglobin is mostly methemoglobin, i.e. all subunits are in their Fe(III) states. Upon exposure to ascorbic acid, a mixed valency species was generated. The $\beta$-subunits were reduced to $\mathrm{Fe}$ (II), while the $\alpha$-subunits remained in their $\mathrm{Fe}$ (III) state. Most probably the Fe(II) subunits were the activating species that abstracted bromine from the initiator and from bromine-terminated polymer chains. This process created a radical and returned the heme to a Fe(III) state. The latter could then transfer bromine back to the radicals.

In addition we studied the related enzyme horseradish peroxidase (HRP) as a catalyst for ATRP. ${ }^{[2 a]}$ It was used to catalyze the polymerization of NIPAAm under ARGET ATRP conditions and yielded bromine-terminated polymers with polydispersity indices as low as 1.44. HRP does not have surface-exposed cysteines that have to be blocked. The stability of the enzyme under the reaction conditions was confirmed by gel electrophoresis, CD spectroscopy, mass spectrometry, and UVvis spectroscopy. Interestingly, the enzyme was found to be in its native resting state ( $\mathrm{Fe}(\mathrm{III}))$ at the beginning and at the end of the polymerization. Therefore it can be concluded that the metal center undergoes redox reactions to $\mathrm{Fe}$ (IV) while involved in the activation step of an ATRP equilibrium.

Independent of our work and in parallel, further proof for the possibility of biocatalytic ATRP was reported by di Lena and coworkers. While the polymerization of PEGMA from the initiator ethyl 2-bromoisobutyrate in the presence of laccase resulted in free radical polymerization, ${ }^{[12]}$ successful ARGET ATRP of PEGA in the presence of BPN was reported using catalase, laccase, and HRP. ${ }^{[13]}$
In a nutshell, these first reports on biocatalytic ATRP clearly show that metalloenzymes can act as catalysts in ATRP and can control radical polymerizations. Thus, we proposed to name this novel activity of promiscuous proteins ATRPase activity. Current work in our laboratory is focused on understanding the underlying biochemical and chemical principles, to further clarify the mechanism of the biocatalytic reaction and to improve and optimize the catalytic performance of ATRPases. A whole toolbox of biotechnological engineering stands open to achieve these goals, including the genetic engineering of biocatalysts and the systematic variation of reaction conditions.

\section{Nanoreactors}

Nanoreactors provide a confined reaction space with dimensions on the nanoscale. ${ }^{14]}$ Chemical reactions are confined to these yoctoliter volumes when catalysts are enclosed in the nanoreactors. Compartmentalization and confinement of reactions into defined reaction spaces is a very common principle in nature. For example, cells and organelles provide microscopic reaction space, while lipid vesicles, the iron-storage protein ferritin, and bacterial protein microcompartments are examples for nanoscale reaction compartments. The organization of reaction cascades into hierarchically ordered reaction compart- ments is key to nature's complex synthetic pathways. In addition, the confinement of reactions into nanoscale reaction volumes offers several advantages for technological applications and synthetic chemistry. For example, side reactions can be suppressed, as the confined volume brings reagent and catalysts into closer spatial proximity. Moreover, the selectivity of a reaction can be altered by controlling the type of substrates to permeate into the nanoreactor. Also, a nanoreactor provides a protective shell that shields the enclosed catalyst from degrading agents outside of the nanoreactor, therefore allowing it to function in hostile environments. Examples for this are non-native enzymatic reactions within living cells. ${ }^{[15]}$ Moreover, nanoreactors can be used to study catalytic reactions on the single molecule level..16] Several types of materials have been investigated as nanoreactors, including mesoporous inorganic materials, ${ }^{[17]}$ nanophase separated polymer networks, ${ }^{[18]}$ or polymersomes. [19] The catalytic species within the confined volume are frequently enzymes. We have been studying two kinds of nanoreactors recently, polymersomes ${ }^{[3 a]}$ and protein cages. ${ }^{[3 b]}$ Both will be highlighted in the next paragraphs.

Amphiphilic block copolymers can self-assemble into vesicles. These nanostructures are often referred to as polymersomes due to their similarity to lipid-based vesicles, the liposomes.[14a] Compared to

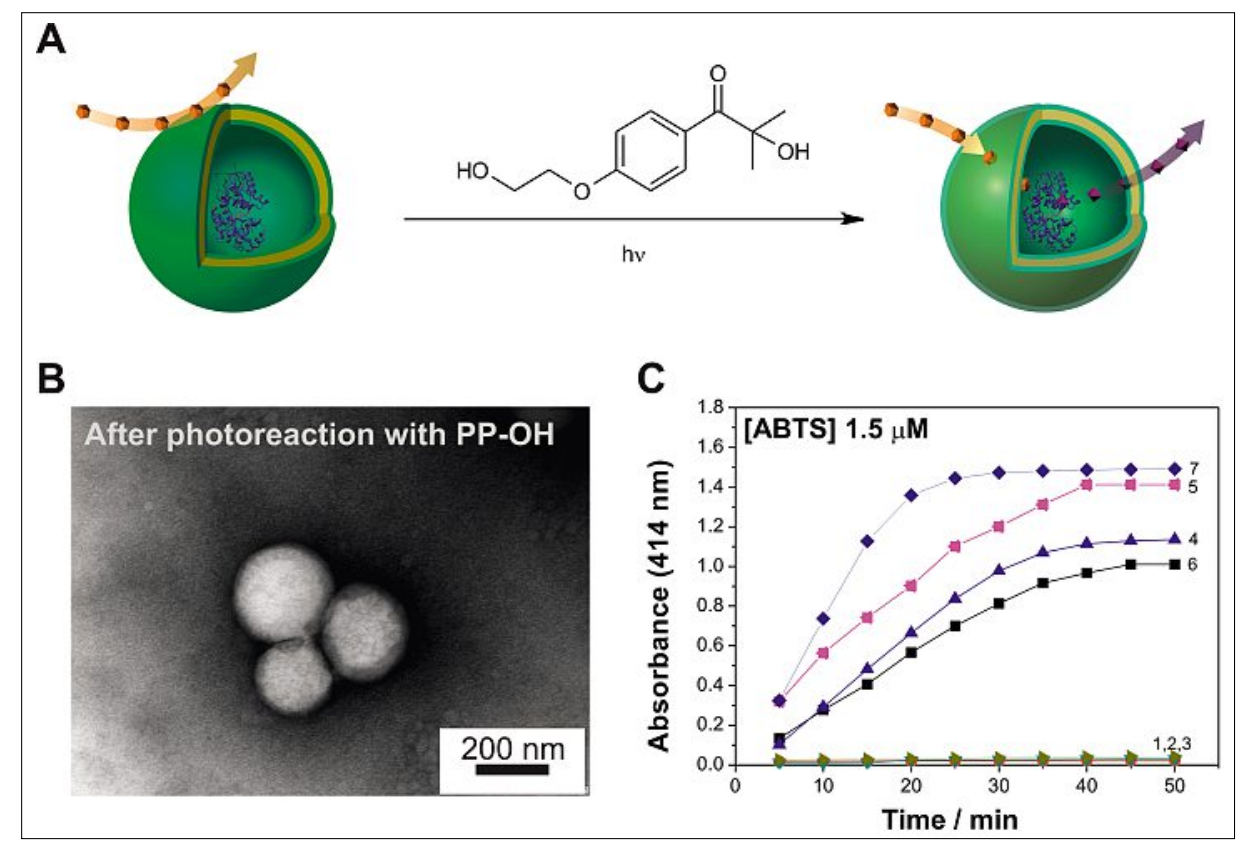

Fig. 2. Photo-permeabilization of polymersomes to yield nanoreactors. A) Horseradish peroxidase (HRP) was encapsulated into polymersomes. They were then made permeable for substrates of the enzyme by photo-reaction with PP-OH. B) TEM micrograph of a HRP-filled polymersome after photo-reaction with PP-OH. C) HRP activity assays with the substrate ABTS to determine the increase in polymersome permeability upon photo-reaction with PP-OH. 1-3) HRP-filled polymersomes before photoreaction with PP-OH; 4-6) HRP-filled polymersomes after photo-reaction with PP-OH 4) A-PMOXA- $b-P D M S-b-P M O X A-A-H R P-P P-O H, 5)$ PMOXA- $b$-PDMS- $b$-PMOXA-HRPPP-OH, 6) PEO- $b$-PB-HRP-PP-OH; 7) free HRP. The initial slope of such kinetic measurements was used to calculate permeabilities. Adapted with permission from ref. [3a]. Copyright 2013 American Chemical Society. 
liposomes, polymeric vesicles are more stable against disintegration, mechanical shearing and storage conditions, and have therefore been investigated extensively as nanocapsules, nanoreactors, and drug-delivery devices. ${ }^{[14 \mathrm{a}, 19 \mathrm{e}, \mathrm{f}]}$ However, most block copolymers form tight membranes that generally do not allow many substances to migrate between the outside and the inside of polymersomes. Mass transport across the membrane is essential for their application as nanoreactors. Therefore, special block copolymers that form an inherently leaky membrane have been synthesized, [20] or the membrane was made permeable for substrates and products by reconstitution of membrane proteins into the block copolymer membranes. ${ }^{[14 a, 15,19 c, f]}$ Recently, we introduced a novel method to permeabilize block copolymers that is not limited to certain types of polymers (Fig. 2A). ${ }^{[3 a]}$ Further, it does not require expensive and difficult to handle membrane proteins. A hydrophilic photo-reactive compound (2-hydroxy-4'-2-(hydroxyethoxy)-2 methylpropiophenone; $\mathrm{PP}-\mathrm{OH})$ was reacted with the membrane of polymersomes under brief irradiation with UV-A light. The membrane was permeabilized for organic molecules because the photo-reagent incorporated into the membrane. The reaction did not alter the shape or size of the polymersomes, as shown by transmission electron microscopy (TEM; Fig. 2B) and light scattering. This treatment preserved the ability of the membrane to retain enzymes within the lumen of the polymersome. The permeabilization was demonstrated with colorimetric substrates for horseradish peroxidase. The enzyme was encapsulated into polymersomes made of poly(2-methyl-2-oxazoline)-blockpoly(dimethylsiloxane)-block-poly(2-

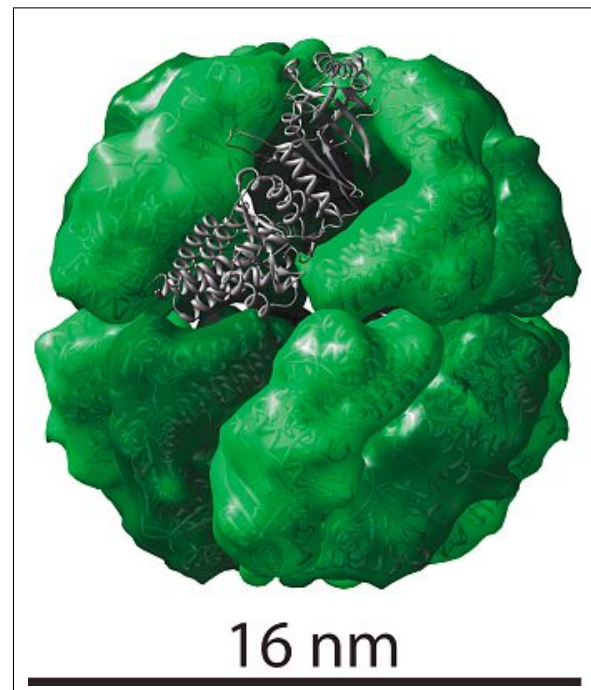

Fig. 3. The protein cage thermosome from Thermoplasma acidophilum is explored as a nanoreactor. Adapted with permission from ref. [14a]. Copyright 2011 Wiley-VCH Verlag GmbH \& Co. KGaA. methyl-2-oxazoline) (PMOXA- $b$-PDMS$b$-PMOXA) and poly(ethylene oxide)block-poly(butadiene) (PEO- $b$-PB) block copolymers. The substrates were not able to access the enzyme through unmodified polymersome membranes (Fig. 2B). However, upon photo-reaction of the polymersomes with PP-OH, the substrates diffused through the membrane into the inside of the vesicles and were converted by HRP into colored products. The permeability of the membrane increased with increasing hydrophobicity of four tested substrates and was measured to be between $1.9 \mathrm{~nm} \mathrm{~s}^{-1}$ for pyrogallol (the most hydrophilic one), $14.4 \mathrm{~nm} \mathrm{~s}^{-1}$ for 2,2'-azino-bis(3-ethylbenzothiazoline-6-sulphonic acid) (ABTS), to $38.2 \mathrm{~nm} \mathrm{~s}^{-1}$ for 3-amino-9-ethyl carbazole (AEC; the most hydrophobic). The PP-OH treated polymersomes were still able to protect the encapsulated peroxidase against degradation by an externally added proteinase. Thus, an easily applicable method was found to render polymersomes semipermeable. This permeabilization method has the potential to convert different types of polymersomes into functional nanoreactors. Such permeabilized nanoreactors could then be applied in technical applications, e.g. as biosensors. Moreover, as HRP possesses ATRPase activity, we currently investigate photopermeabilized polymersomes as nanoreactors for biocatalytic ATRP.

An intriguing possibility for responsive nanoreactors is the use of protein cages. Examples are ferritin, ${ }^{[21]}$ viral capsids, ${ }^{[16,22]}$ and bacterial microcompartments. ${ }^{23]}$ These hollow nanostructures self-assemble from various protein subunits, and range in diameter from $<10$ $\mathrm{nm}$ to more than $500 \mathrm{~nm}$. Some of them assemble or disassemble in response to changes in their environment ${ }^{[16]}$ and others possess pores that open, widen or close in the presence of certain triggers. ${ }^{[24]}$ We have explored the thermosome, a chaperonin from the archea Thermoplasma acidophilum, as a functional nanostructure (Fig. 3). For example, we have entrapped a pair of fluorescent proteins and used the thermosome as a scaffold that brings the proteins into defined proximity to enable fluorescence resonance energy transfer (FRET) between the two proteins. ${ }^{[25]}$ More recently, we have focused on the possibility to use the thermosome as an adenosine triphosphate (ATP) -triggered nanoreactor for polymerization reactions, ${ }^{[3 b]}$ as it has gated pores that are large enough to allow macromolecules and synthetic polymers to enter and leave the protein cage. These pores can open and close in response to ATP and its analogues. We have encapsulated a copper complex as catalysts for ATRP into the cavity of the thermosome and conducted ATRP of NIPAAm and other monomers within the cage. The effect of the confined reaction space manifested itself in smaller but more narrowly dispersed polymer products as compared to polymers synthesized under comparable conditions without a protein nanoreactor.

\section{Damage Self-reporting Polymer Composites}

Fiber-reinforced composites are lightweight, yet strong polymeric materials and find use in aerospace applications, the automotive sector, in sports equipment, and many other high performance fields. However, they are prone to microdamages caused by the impact of objects, ${ }^{[26]}$ e.g. a dropped tool during manufacture or maintenance. These microdamages manifest themselves as de-

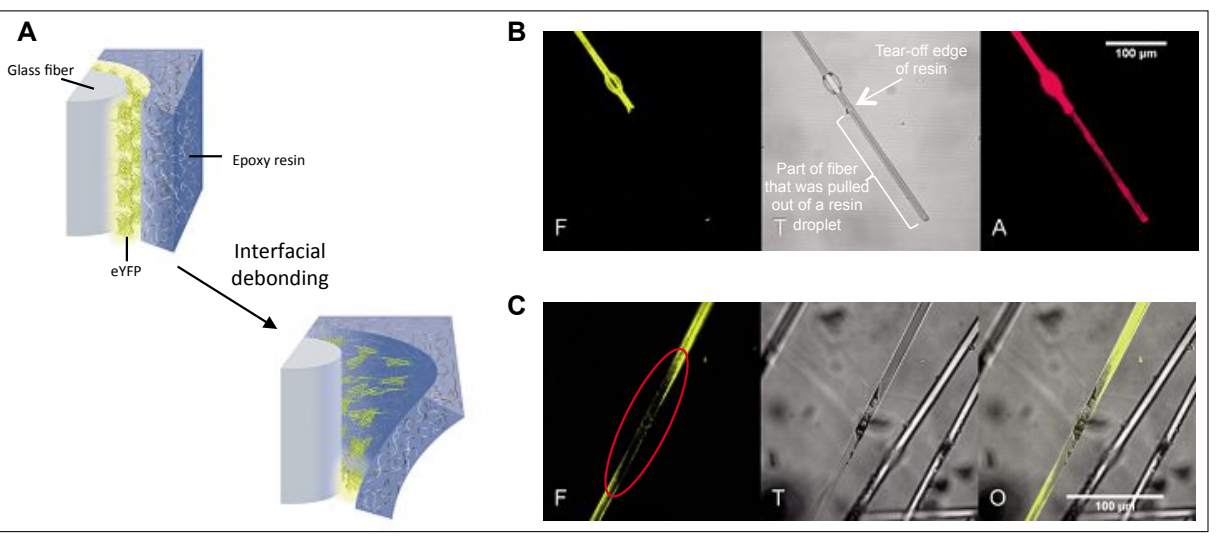

Fig. 4. A) Fluorescent protein acting as a mechanophore at the fiber-resin interface in fiber-reinforced composites. Interfacial debonding defects cause mechanical unfolding of the protein and thereby a loss of the protein's fluorescence. B) Confocal fluorescence image of eYFP on a glass fiber after fiber pullout from a microdroplet of epoxy resin. The fiber was immunostained with an Alexa Fluor 647-antibody in order to detect native and unfolded eYFP. (Channels: $F=e Y F P, T=$ transmission, A = Alexa Fluor 647); C) Confocal microscopy image of an eYFP/glass fiber/epoxy resin composite after having been subjected to low velocity impact. Diminished fluorescence in the vicinity of a fiber fracture is marked by a red oval. (Channels: $\mathrm{F}=$ eYFP, $\mathrm{T}=$ transmission, $\mathrm{O}$ = overlay) Adapted with permission from ref. [4]. Copyright 2013 Wiley-VCH Verlag GmbH \& Co. KGaA. 
lamination of fiber plys, as debonding of the polymer resin from reinforcing fibers, or as fiber fracture. Microdamages can initiate large-scale damage when the material is under mechanical stress. Therefore, detection of damages is essential in order to replace or repair the damaged material before catastrophic material failure occurs. Unfortunately, these micro damages are usually barely visible. Conventional methods to detect them are based on nondestructive evaluation techniques, such as X-ray, ultrasound or thermography. ${ }^{[27]} \mathrm{A}$ possibility to detect damage at even smaller scales, i.e. on the molecular scale, is to embed molecules that react to mechanical stress with a change in their spectral properties.[28] Fluorescent proteins only fluoresce if their native structure is intact, and they can be denatured by mechanical forces. ${ }^{[29]}$ Thus, a transition from a fluorescent to non-fluorescent state can indicate stress. We recently showed that fluorescent proteins can be mechanically unfolded at the interface of glass fibers and polymer resin in fiber-reinforced composites (Fig. $4 \mathrm{~A}) .{ }^{[4]}$ Glass fibers were modified with an aminosilane layer and enhanced yellow fluorescent protein (eYFP) was covalently bound to the fibers by means of the linker disuccinimidyl terephthalate. The functionalized fibers were embedded into a microdroplet of epoxy resin and then pulled out of the resin using a micromechanical test apparatus. The fibers lost their yellow fluorescence in the contact zone between resin droplet and fiber (Fig. 4B). eYFP was, however, still present in this area, as shown by immunostaining with an antibody that binds to native and denatured eYFP. It can be concluded that the fluorescence of the protein was switched off due to the debonding of the resin droplet from the fiber. The same effect could be observed in composite materials as a response to low velocity impacts perpendicular to the fibers. Fibers that were embedded into epoxy resin fluoresced in intact areas of the composite materials, but lost their fluorescence in the vicinity of fiber fractures, i.e. in zones of fiber-resin debonding (Fig. 4C). Such changes in the fluorescence can easily be detected by optical means and could be useful as a frontline detection of microdamages. Moreover, the proteins could be used as force probes for the investigation of the mechanisms of damage propagation at the fiber-resin interface. Interestingly, this work represents the first example of exploiting the mechanophoric properties of fluorescent proteins in a material. Moreover, it is one of the few examples in which external forces alter the properties of proteins in a polymer. Materials with embedded mechanoresponsive proteins and enzymes have been proposed to be useful for catalytic switches and as sensing devices. The reader is referred to an excellent current review on mechanobiochemistry for further inspiration. ${ }^{[30]}$

\section{Conclusions}

Proteins are a very versatile class of macromolecules within the broad variety of functional molecules in nature. When enzymes are used to synthesize polymers, or when proteins are combined with synthetic polymers, interesting new routes towards environmentally friendly catalysis, functional materials or functional nanosystems arise. The examples highlighted in this review cover catalytic activities of classic enzymes such as horseradish peroxidase and hemoglobin, ATP-responsive protein cages, and the mechanoresponsive properties of fluorescent proteins. While this is only an arbitrary selection of functions that proteins have to offer, it shows the huge potential that can be unlocked by combining polymer sciences with protein sciences.

\section{Acknowledgements}

We thank Prof. Wolfgang Meier (University of Basel) for his tremendous support over the last years. Financial support by the Swiss National Science Foundation, the NCCR Nanoscale Sciences, the Holcim Stiftung Wissen, the Marie Curie Actions of the European Commission, the KTI/CTI, and Sciex-NMS ${ }^{\text {ch }}$ is gratefully acknowledged.

Received: July 10, 2013

[1] a) M. D. Hager, P. Greil, C. Leyens, S. van der Zwaag, U. S. Schubert, Adv. Mater. 2010, 22, 5424; b) E. B. Murphy, F. Wudl, Prog. Polym. Sci. 2010, 35, 223.

[2] a) S. J. Sigg, F. Seidi, K. Renggli, T. B. Silva, G. Kali, N. Bruns, Macromol. Rapid Commun. 2011, 32, 1710; b) G. Kali, T. B Silva, S. J. Sigg, F. Seidi, K. Renggli, N. Bruns, in 'Progress in Controlled Radical Polymerization: Mechanisms and Techniques', ACS Symposium Series, Vol. 1100, American Chemical Society, 2012, pp. 171; c) T. B. Silva, M. Spulber, M. K. Kocik, F. Seidi, H. Charan, M. Rother, S. J. Sigg, K. Renggli, G. Kali, N. Bruns, Biomacromolecules 2013, 14, 2703.

[3] a) M. Spulber, A. Najer, K. Winkelbach, O. Glaied, M. Waser, U. Pieles, W. Meier, N. Bruns, J. Am. Chem. Soc. 2013, 135, 9204; b) K. Renggli, M. G. Nussbaumer, R. Urbani, T Pfohl, N. Bruns, Angew. Chem. Int. Ed. 2013, DOI: $10.1002 / a n i e .201306798$, and DOI: 10.1002/ange. 201306798.

[4] K. Makyła, C. Müller, S. Lörcher, T. Winkler, M. G. Nussbaumer, M. Eder, N. Bruns, $A d v$. Mater. 2013, 25, 2701

[5] a) W. A. Braunecker, K. Matyjaszewski, Prog. Polym. Sci. 2007, 32, 93; b) N. V. Tsarevsky, K. Matyjaszewski, Chem. Rev. 2007, 107, 2270; c) K. Matyjaszewski, Macromolecules 2012, 45, 4015.

[6] a) 'Biocatalysis in Polymer Chemistry', Ed. K. Loos, Wiley-VCH, Weinheim, Germany, 2010 b) S. Kobayashi, A. Makino, Chem. Rev. 2009, 109, 5288; c) F. Hollmann, I. W. C. E. Arends, Polymers 2012, 4, 759

7] K.-H. van Pée, S. Unversucht, Chemosphere 2003, 52, 299.
[8] a) M. Brunori, Trends Biochem. Sci. 1999, 24, 158; b) K. Imai, Nature 1999, 401, 437.

[9] a) B. J. Reeder, Antioxid. Redox Signaling 2010, 13, 1087; b) O. V. Kosmachevskaya, A. F. Topunov, Appl. Biochem. Microbiol. 2009, 45, 563.

[10] a) A. M. Azevedo, V. C. Martins, D. M. F. Prazeres, V. Vojinovic, J. M. S. Cabral, L. P. Fonseca in 'Biotechnology Annual Review', vol. 9, Ed. M. R. El-Gewely, Elsevier, 2003, pp. 199; b) N. C. Veitch, Phytochemistry 2004, 65, 249.

[11] P. Walde, Z. Guo, Soft Matter 2011, 7, 316.

[12] Y.-H. Ng, F. di Lena, C. L. L. Chai, Polym. Chem. 2011, 2, 589.

[13] Y.-H. Ng, F. di Lena, C. L. L. Chai, Chem. Commun. 2011, 47, 6464

[14] a) K. Renggli, P. Baumann, K. Langowska, O. Onaca, N. Bruns, W. Meier, Adv. Funct. Mater. 2011, 21, 1241; b) K. T. Kim, S. A. Meeuwissen, R. J. Nolte, J. C. van Hest, Nanoscale 2010, 2, 844.

[15] P. Tanner, V. Balasubramanian, C. G. Palivan, Nano Lett. 2013, 13, 2875.

[16] M. Comellas-Aragones, H. Engelkamp, V. I. Claessen, N. A. J. M. Sommerdijk, A. E. Rowan, P. C. M. Christianen, J. C. Maan, B. J. M. Verduin, J. J. L. M. Cornelissen, R. J. M. Nolte, Nat. Nanotechnol. 2007, 2, 635.

[17] Y. Masuda, S.-i. Kugimiya, K. Murai, A. Hayashi, K. Kato, Colloids Surf., B 2013, 101, 26.

[18] N. Bruns, J. C. Tiller, Nano Lett. 2005, 5, 45.

[19] a) J. Gaitzsch, D. Appelhans, L. Wang, G. Battaglia, B. Voit, Angew. Chem., Int. Ed. 2012, 51, 4448; b) M. Marguet, C. Bonduelle, S. Lecommandoux, Chem. Soc. Rev. 2013, 42 , 512; c) C. Nardin, S. Thoeni, J. Widmer, M. Winterhalter, W. Meier, Chem. Commun. 2000, 1433; d) K. Langowska, C. G. Palivan, W. Meier, Chem. Commun. 2013, 49, 128; e) R. J. R. W. Peters, I. Louzao, J. C. M. van Hest, Chem. Sci. 2012, 3, 335; f) P. Tanner, P. Baumann, R. Enea, O. Onaca, C. Palivan, W. Meier, Acc. Chem. Res. 2011, 44, 1039.

[20] a) S. M. Kuiper, M. Nallani, D. M. Vriezema, J. J. L. M. Cornelissen, J. C. M. van Hest, R. J. M. Nolte, A. E. Rowan, Org. Biomol. Chem. 2008, 6, 4315; b) H.-P. M. de Hoog, D. M. Vriezema, M. Nallani, S. Kuiper, J. J. L. M. Cornelissen, A. E. Rowan, R. J. M. Nolte, Soft Matter 2008, 4, 1003 ; c) K. T. Kim, M. A. Winnik, I. Manners, Soft Matter 2006, 2, 957.

[21] T. Ueno, M. Suzuki, T. Goto, T. Matsumoto, K. Nagayama, Y. Watanabe, Angew. Chem., Int. Ed. 2004, 43, 2527

[22] D. P. Patterson, P. E. Prevelige, T. Douglas, ACS Nano 2012, 6, 5000 .

[23] T. O. Yeates, C. A. Kerfeld, S. Heinhorst, G. C. Cannon, J. M. Shively, Nat. Rev. Microbiol. 2008, 6, 681

[24] a) J. A. Speir, S. Munshi, G. Wang, T. S. Baker, J. E. Johnson, Structure 1995, 3, 63; b) M. G. Bigotti, A. R. Clarke, Arch. Biochem. Biophys. 2008, 474, 331.

[25] a) N. Bruns, K. Pustelny, L. M. Bergeron, T. A. Whitehead, D. S. Clark, Angew. Chem., Int. Ed. 2009, 48, 5666; b) N. Bruns, D. S. Clark, Chimia 2011, 65, 245.

[26] J. W. C. Pang, I. P. Bond, Compos. Sci. Technol. 2005, 65, 1791

[27] X. E. Gros, in 'Handbook of polymer testing: physical methods', Ed.: R. Brown, Marcel Dekker, New York, 1999, pp. 773

[28] a) M. M. Caruso, D. A. Davis, Q. Shen, S. A. Odom, N. R. Sottos, S. R. White, J. S. Moore, Chem. Rev. 2009, 109, 5755; b) A. L. Black, J. M. Lenhardt, S. L. Craig, J. Mater. Chem. 2011, $21,1655$.

[29] a) H. Dietz, M. Rief, Proc. Natl. Acad. Sci. USA 2004, 101, 16192; b) M. Caraglio, A. Imparato, A. Pelizzola, Phys. Rev. E: Stat., Nonlinear, Soft Matter Phys. 2011, 84, 021918

[30] J. N. Brantley, C. B. Bailey, K. M. Wiggins, A. T. Keatinge-Clay, C. W. Bielawski, Polym. Chem. 2013, 4, 3916. 